\title{
The Staffordshire Arthritis, Musculoskeletal, and Back Assessment (SAMBA) Study: a prospective observational study of patient outcome following referral to a primary-secondary care musculoskeletal interface service
}

\author{
Edward Roddy ${ }^{* 1,2}$, Irena Zwierska1, Peter Dawes², Samantha L Hider ${ }^{1,2}$, Kelvin P Jordan1, Jon Packham¹,2,
}

Kay Stevenson 2,3, Elaine Hay 1,2 and the SAMBA team

\begin{abstract}
Background: Recent healthcare policy has shifted the management of musculoskeletal conditions in the UK away from secondary care towards Clinical Assessment and Treatment Services at the primary-secondary care interface. However, little is known about the outcome of patients with musculoskeletal conditions referred from primary care to Clinical Assessment and Treatment Services or how best to identify those patients at high risk of poor outcome in this setting. We describe the protocol for a twelve-month prospective observational study which aims to describe the outcome of patients referred to musculoskeletal and back pain services at the primary-secondary care interface and to develop simple prognostic measures to guide clinical prioritisation and triage.

Methods/Design: All patients referred over a twelve-month period from primary care to musculoskeletal and back pain clinics in the primary-secondary care interface Clinical Assessment and Treatment Service in North Staffordshire will be mailed a postal questionnaire prior to their consultation. This will collect information on quality of life, general health, anxiety and depression, pain, healthcare utilisation including medication use, occupational characteristics, and socio-demographics. At the consultation in the interface clinic, the clinical diagnosis, investigations requested, and clinical interventions will be recorded. Follow-up data for the twelve-month period subsequent to recruitment will be collected via mailed follow-up questionnaires at 6 and 12 months, and review of medical records.

Discussion: This twelve-month prospective observational study of patients referred to a musculoskeletal Clinical Assessment and Treatment Service will assess the management and outcome of musculoskeletal care at the primarysecondary care interface as proposed in the Musculoskeletal Services Framework.
\end{abstract}

\section{Background}

Musculoskeletal conditions such as back pain, osteoarthritis and regional pain are highly prevalent and associated with a considerable burden of pain, disability and work loss [1]. It is estimated that one in five adults will consult primary care for a musculoskeletal problem during a one-year period [2]. Most non-inflammatory mus-

* Correspondence: e.roddy@cphc.keele.ac.uk

${ }^{1}$ Arthritis Research Campaign National Primary Care Centre, Primary Care Sciences, Keele University, Staffordshire, ST5 5BG, UK

Full list of author information is available at the end of the article culoskeletal disorders are managed in primary care. Traditionally, referral to secondary care has been to specialist services such as orthopaedics or rheumatology, in a setting supported by the multidisciplinary team. Whilst certain patients referred to secondary care require care in that setting, particularly those deemed suitable for joint replacement surgery, many patients can be managed appropriately at the interface between primary and secondary care. The recently published Musculoskeletal Services Framework [3] and UK Government White Paper "Our Health, Our Care, Our Say" [4] outline a change in 
emphasis shifting the management of musculoskeletal conditions in the UK away from secondary care towards Clinical Assessment and Treatment Services (CATS) at the primary-secondary care interface. This change aims to improve efficiency, provide treatment closer to patients' homes, and, with effective triage, reduce inappropriate referrals to secondary care services such as rheumatology and, in particular, orthopaedics [5]. The generic functions of CATS are to provide an expert multidisciplinary opinion, screen for important remedial conditions and refer such conditions appropriately, direct patients to appropriate services for investigation, agree evidence-based integrated care pathways, facilitate referral to other primary and secondary care services where necessary, and support the development of robust systems for monitoring and clinical audit [3].

The Musculoskeletal Services Framework identified several key issues with traditional referral pathways from primary to secondary care, including long waiting times for assessment and treatment which are unacceptable to patients [6] and may adversely influence outcome $[7,8]$, and a lack of clear integrated care pathways [3]. It attempts to tackle these issues by proposing the development of pathways which manage patient flows through primary/secondary care to ensure appropriate and timely referral to specialist care services and use capacity in acute settings appropriately. Effective triage to ensure patient referrals from primary care reach the most appropriate destination is a key component of such pathways. Traditionally, both the triage destination and clinical priority have been determined by the information provided in the referral letter. However, many referrals to hospitalbased musculoskeletal services are misdirected and provide insufficient clinical information to guide triage [9]. The Musculoskeletal Services Framework has proposed use of tools to guide clinical triage and prioritise urgency of surgery [10-12]. However, existing measures are jointspecific and have tended to focus on surgical priority in secondary care. There is a need for generic clinical priority measures, which can be used to guide clinical triage across a wider range of musculoskeletal conditions at the primary-secondary care interface.

Musculoskeletal conditions which might previously have been managed in secondary care will be increasingly managed at the primary-secondary interface. However, there is a paucity of evidence on the transition of patients from primary to secondary care and outcome of patients with musculoskeletal conditions referred from primary care to CATS. The natural history, progression and outcome in patients with a range of musculoskeletal conditions have been described in primary care [13-17] and secondary care (i.e. rheumatology) [18-20] settings. A small number of studies have examined the outcome of GP referrals to secondary care (including orthopaedic and rheumatology clinics) and have highlighted issues such as high rates of re-attendance [21], the poor outcome of many patients [22,23] and the role of patient pressure in the referral process [24]. These studies have tended to focus on a single or limited number of disease areas, for example, knee osteoarthritis, shoulder pain or rheumatoid arthritis.

We describe here the protocol for a twelve-month prospective, observational cohort study which aims, firstly, to describe the characteristics, management, and clinical and health-economic outcomes, of patients with a wide range of musculoskeletal conditions referred from primary care to an established musculoskeletal interface CATS, and, secondly, to develop simple prognostic measures for use as clinical triage tools to identify patients at high risk of poor outcome from musculoskeletal conditions and back pain at the primary-secondary interface (Figure 1).

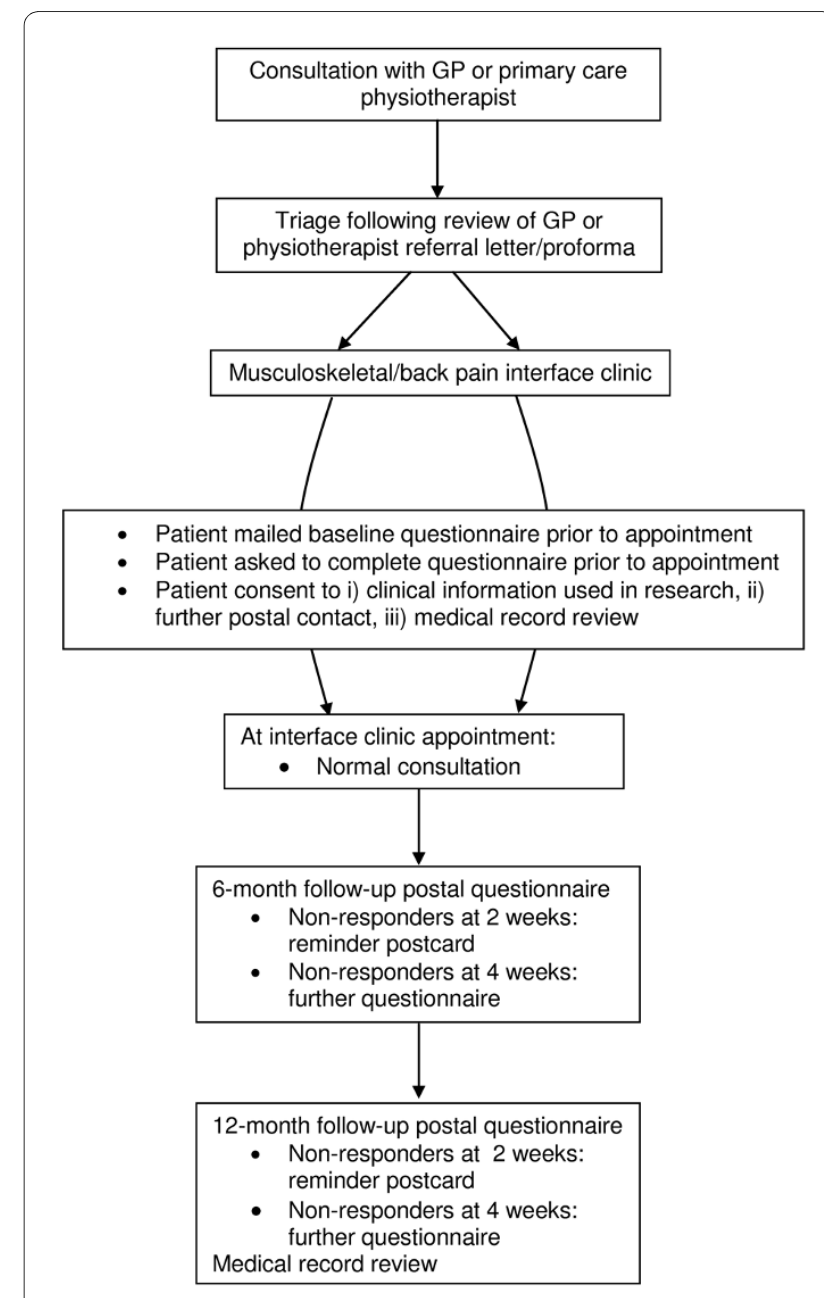

GP, General practitioner

Figure 1 Flow diagram outlining participant's study pathway 


\section{Methods/design Design}

The study will be a twelve-month prospective observational study undertaken in the setting of musculoskeletal interface services in North Staffordshire. Ethical approval for the study has been obtained from South Staffordshire Local Research Ethics Committee (REC reference number: 07/H1203/86).

\section{Study setting and population}

Musculoskeletal interface services in North Staffordshire were highlighted in the Musculoskeletal Services Framework as a successful model of interface care which incorporate dynamic triage of pooled musculoskeletal referrals and clinical algorithms for a wide range of conditions. CATS clinics are located in local hospitals, health centres and general practices [3]. These CATS clinics utilise multidisciplinary staffing including rheumatologists, consultant physiotherapists, extended scope physiotherapists and GPs with special interests. The service has reduced waiting times in accordance with current NHS targets, increased orthopaedic conversion rates and been highly rated in satisfaction surveys of patients and GPs [3].

The study population will consist of all patients referred from primary care and subsequently triaged to musculoskeletal and back pain interface clinics in Stoke-on-Trent Primary Care Trust (PCT) over a twelve-month period. All adults aged 18 years and over and able to understand and capable of giving written informed consent will be considered eligible to participate in the study.

\section{Initial contact}

Referral letters for all patients with musculoskeletal problems will be triaged by the clinical team according to usual clinical care. Those patients triaged to the CATS will be potentially eligible for inclusion in the study and will be sent a study pack two weeks prior to their appointment containing a letter of invitation, study participant information sheet and baseline self-administered questionnaire consisting of validated health assessment instruments. The appointments process will generate a study database to facilitate mailing of study packs directly to patients. Patients will be asked to complete the baseline questionnaire prior to their appointment but will also be advised that the study will be explained to them again when they attend for the clinic appointment, so that they can ask any questions they wish at that time. Patients will be informed that there is no obligation for them to participate if they do not wish to do so, and if they decline they will continue to receive normal clinical care. Patients willing to participate will be asked to provide full written informed consent. The last page of the questionnaire will include three consent questions; (1) for use of clinical information taken at their initial clinic visit to be used in the research, (2) for further contact by postal questionnaire; (3) for review of medical records.

\section{Baseline questionnaire}

Details of the information to be collected by the baseline questionnaire are presented in Table 1 . This includes validated quality of life, general health, musculoskeletalrelated, demographic, occupational and health care use scales. It will also include three potential prognostic tools: the modified Salisbury score is a clinical priority score used in routine clinical practice in Stoke-on-Trent (table 2) $[25,26]$. We have developed a patient-completed version of the Salisbury score, which we aim to validate in this study. The PROG-RES [27] and StarT Back tools [28] have been developed by our centre with the aims of identifying patients at high risk of poor outcome from musculoskeletal conditions and back pain in primary care.

\section{Clinic appointment}

When they attend for their routine clinic appointment, patients will be seen by a research assistant who will explain the study again, answer any questions the patient may have, and check the questionnaire and consent form for completeness. The clinical diagnosis, investigation(s) requested, intervention(s), and plan for clinical follow-up will be recorded on a standard paper proforma by the interface clinician conducting the clinical consultation. The interface clinician will also be asked to complete the modified Salisbury score (Table 2). This score is also routinely completed by the referring GP or physiotherapist at the time of referral to musculoskeletal services at the primary-secondary care interface. The referrer-completed modified Salisbury score will be transposed from the referral letter (where completed).

\section{Pilot of baseline procedure}

The self-report questionnaire will be piloted in a small number of patients attending two musculoskeletal clinics to assess its acceptability to patients and ease of completion (approximately 5-10 patients). The processes for recruitment and obtaining consent will be piloted in one back pain and one musculoskeletal clinic per week over a one-month period (approximately 100 patients). The clinician paper proforma was developed in consultation with musculoskeletal interface clinicians and piloted in CATS clinics.

\section{Follow-up questionnaires}

A self-administered questionnaire will be mailed to all participants at 6 and 12 months. Non-responders will be sent a postcard reminder after 2 weeks and a repeat questionnaire after 4 weeks. The 6- and 12-month follow-up questionnaires will be identical to the baseline questionnaire (Table 1). Participants will also be asked to rate (1) 


\section{Table 1: Content of the baseline postal questionnaire}

\begin{tabular}{|c|c|c|}
\hline Concept & Measurement method & Detail \\
\hline Quality of life & EuroQOL (EQ-5D) [31] & Five dimension descriptive system (today) \\
\hline Perceived general health & MOS Short Form-36 (SF-36) version 2 [32] & $\begin{array}{l}\text { 36-items covering eight health domains } \\
\text { (past } 4 \text { weeks) }\end{array}$ \\
\hline \multirow[t]{2}{*}{ Anxiety and depression } & Hospital anxiety and depression scale [33] & $\begin{array}{l}\text { Anxiety and depression sub-scales (past } \\
\text { week) }\end{array}$ \\
\hline & $\begin{array}{l}\text { Depression Screening questions for } \\
\text { primary care [34] }\end{array}$ & Low mood/anhedonia in the last month \\
\hline \multirow[t]{3}{*}{ Bodily pain } & Location: Self-completed manikin & $\begin{array}{l}\text { "In the past } 4 \text { weeks, have you had pain } \\
\text { that has lasted for one day or longer in any } \\
\text { part of your body?" }\end{array}$ \\
\hline & Current pain severity [35] & $\begin{array}{l}\text { 0-10 NRS with verbal anchors (no pain, } \\
\text { pain as bad as can be) }\end{array}$ \\
\hline & Episode duration [36] & $\begin{array}{l}<3 \text { months, } 3-6 \text { months, } 7-12 \text { months, } 1-2 \\
\text { years, 3-5 years, } 6-10 \text { years, } 10+\text { years }\end{array}$ \\
\hline \multirow[t]{3}{*}{ Clinical triage and prioritisation tools } & Patient-completed Salisbury score & $\begin{array}{l}\text { 6-item triage tool for musculoskeletal } \\
\text { problems referred from primary care }\end{array}$ \\
\hline & PROG-RES tool [27] & $\begin{array}{l}\text { 7-item prognostic tool for musculoskeletal } \\
\text { pain in primary care }\end{array}$ \\
\hline & Modified STarT Back screening tool [28] & $\begin{array}{l}\text { 7-item modified screening tool for } \\
\text { musculoskeletal prognostic indicators } \\
\text { including } 5 \text {-item psychosocial subscale } \\
\text { (past } 2 \text { weeks) }\end{array}$ \\
\hline Knee pain & $\begin{array}{l}\text { Western Ontario and McMaster } \\
\text { Universities Osteoarthritis index (WOMAC) } \\
\text { [37] }\end{array}$ & $\begin{array}{l}\text { Pain }(0-20) \text {, stiffness }(0-8), \text { physical } \\
\text { functioning }(0-68)\end{array}$ \\
\hline \multirow{2}{*}{$\begin{array}{l}\text { Healthcare utilisation for musculoskeletal } \\
\text { problems }\end{array}$} & Investigations/treatment & \\
\hline & Medication use & $\begin{array}{l}\text { Analgesics, NSAIDs, opiates, natural } \\
\text { remedies, glucosamine, chondroitin }\end{array}$ \\
\hline \multirow[t]{6}{*}{ Occupational characteristics } & $\begin{array}{l}\text { Job title/type of work for most of working } \\
\text { life }\end{array}$ & \\
\hline & Current employment status & $\begin{array}{l}\text { Working full-time/part-time, Employed } \\
\text { but off-sick for }<6 \text { months, Looking after } \\
\text { home/children, Not working for }>6 \\
\text { months due to joint/back problem, Fully } \\
\text { retired, Early retirement due to joint/back } \\
\text { problem, Student }\end{array}$ \\
\hline & $\begin{array}{l}\text { Work absence during last } 6 \text { months due to } \\
\text { joint/back problems }\end{array}$ & Yes/No \\
\hline & Current work status & $\begin{array}{l}\text { Doing usual job, working fewer hours, } \\
\text { doing lighter duties, paid/unpaid sick } \\
\text { leave }\end{array}$ \\
\hline & Stanford presenteeism scale (SPS-6) [38] & $\begin{array}{l}\text { 6-items concerning Health Status and } \\
\text { Employee Productivity (5-point Likert } \\
\text { scale) }\end{array}$ \\
\hline & $\begin{array}{l}\text { Effect of joint/back problems on } \\
\text { productivity }\end{array}$ & $\begin{array}{l}\text { 0-10 NRS with verbal anchors (no effect, } \\
\text { completely prevented me from working) }\end{array}$ \\
\hline
\end{tabular}


Table 1: Content of the baseline postal questionnaire (Continued)

\begin{tabular}{lll}
\hline Demographic characteristics & Date of birth, gender & Married, separated, divorced, widowed, \\
& Marital status & $\begin{array}{l}\text { Alone, not alone } \\
\text { cohiting, single }\end{array}$ \\
Anthropometric data & Living arrangements & \\
Lifestyle characteristics & Self-reported height & \\
& Smoking status & Never, previously, currently \\
& Alcohol intake & Daily, weekly, monthly, yearly, never \\
\hline
\end{tabular}

their satisfaction with the care they have received on a 5point Likert scale ranging from "Very satisfied" to "Not at all satisfied" and (2) how their musculoskeletal problem compares to 6 months previously on a 5-point Likert scale ranging from "Much better" to "Much worse".

\section{Medical record review}

Medical record review from baseline to 12 months will seek to identify: (1) relevant comorbidities, (2) repeat consultation in primary care with the same musculoskeletal problem (clinician-recorded diagnosis mapped to standard Read morbidity codes [29]) and, (3) further referral to interface clinics or secondary care.

\section{Sample size}

Approximately 3500 patients are seen in the musculoskeletal and back pain interface clinics in North Staffordshire during the course of a year. Based on previous studies, we would expect $75 \%$ of these to participate in the baseline stage $(n=2625)$. Based on previous work within our Centre [30], we would expect $75 \%$ of these to consent to further contact and $75 \%$ to consent to medical record review. This would mean around 2000 participants having their medical records reviewed. Given a $75 \%$ response to each follow-up questionnaire, then around 1500 people would return 6-month questionnaires and 1125 people 12-month questionnaires. A sample size of 1125 is sufficient to determine the percentage referred to the interface clinics who make a repeat consultation to primary care during the course of the following year with a margin of error of 3\% and a 95\% confidence level, based on an actual estimate of $50 \%$.

\section{Analysis}

The primary analysis will be simple descriptive statistics (frequencies, percentages, means, medians where appropriate with confidence intervals) of the characteristics of patients seen in interface clinics at baseline, and the num-

\section{Table 2: Modified Salisbury score completed by referring GP/physiotherapist and interface-clinician}

Please rate your assessment of the patient's current pain/problems:

1. How do you rate the progress of the problem?

Stable/improving (0)

Slowly worsening (months) (1)

Worsening steadily (weeks) (2)

Rapidly worsening (days) (3)

4. How do you rate the loss of physical function?
2. How do you rate the pain that the patient is experiencing?

No pain (0)

Occasional pain (1)

Frequent pain (2)

Constant pain (night \& day) (3)

5. How do you rate the patient's dependence on others?
3. How do you rate the distress that the patient is experiencing (psychosocial)?

None (no worry) (0)

Mild (occasional worry) (1)

Moderate (frequently worried (2)

Severe (constant distraction) (3)

6. How do you rate the specific effect on the patient's ability to perform normal activities during the last week (ie social, housework, educational, recreational)?

Not affected (0)

Coping but affected (1)

Not coping some days ( $<3$ days) (2)

Total incapacity (3)
$0-25 \%$ loss of function (0)
$26-50 \%$ loss of function (1)
$51-75 \%$ loss of function (2)
$76-100 \%$ loss of function (3)

No dependence $(0)$

Regular help needed (2)

Substantial dependence (3) 
ber of repeat consultations to primary care over the twelve-month period. Changes within outcome measures (e.g. EuroQOL, Short Form-36 (SF-36), Hospital Anxiety and Depression Scale) over the duration of the study will also be examined using paired $t$-tests for continuous data and McNemar tests for dichotomous data.

Secondary analyses will involve comparisons between different patient groups (depending on the case-mix seen) and to determine baseline factors predicting change on outcome measures over the course of the study and clinical response to specific interventions. Multiple linear regression will be used for continuous outcomes and logistic regression for dichotomous outcomes.

The three modified Salisbury scores (referrer-completed, musculoskeletal clinician-completed and patientcompleted) will be compared to each other at baseline using intraclass correlation coefficients. Each modified Salisbury score will be compared to the primary care musculoskeletal assessment tool [27] and STarT Back screening tool [28] using Pearson's correlation coefficient at baseline. The ability of each to predict repeat consultation, global change and changes within other health status measures (e.g. SF-36) over the duration of the study will be examined by comparing those with predicted poor outcomes to those with better prognoses on the prognostic tools. Sensitivity and specificity measures will be used to assess prognostic ability compared to the outcome measures.

\section{Discussion}

Recent UK healthcare policy has advocated providing non-surgical care of common musculoskeletal conditions such as back pain, osteoarthritis and regional pain in CATS at the primary-secondary care interface [3]. However, a paucity of research evidence exists to support this policy and the clinical and cost-effectiveness of such interface services is not known. This protocol describes a twelve-month prospective observational study of the characteristics, management and outcome of patients referred to a musculoskeletal CATS at the primary-secondary interface in North Staffordshire. The study will assess the effectiveness of musculoskeletal care at the primary-secondary care interface, determine simple tools to guide prioritisation, and potentially influence the design of future care pathways.

\section{Competing interests}

The authors declare that they have no competing interests.

\section{Authors' contributions}

All authors participated in the design of the study, acquisition of data, and drafting of the manuscript. All authors read and approved the final manuscript.

\section{Acknowledgements}

This work is supported by an Arthritis Research Campaign Integrated Clinical Arthritis Centre Grant (17684), the Arthritis Research Campaign National Primary Care Centre Grant (18139), funding secured from Stoke-on-Trent Primary
Care Trust (PCT), and service support through the West Midlands North CLRN. The authors would like to thank the administrative and health informatics staff and research nurse teams at the Arthritis Research Campaign National Primary Care Centre and Haywood Hospital, and the clinicians, staff and patients of Stoke PCT musculoskeletal and back pain interface services. SAMBA team members: Joanne Bailey, Helen Duffy, Tina Gilbert, Rhian Hughes, Zoë Mayson, Janet Ough, Diane Stanyer, Vicki Taylor, Sue Weir.

\section{Author Details}

${ }^{1}$ Arthritis Research Campaign National Primary Care Centre, Primary Care Sciences, Keele University, Staffordshire, ST5 5BG, UK, 2Staffordshire Rheumatology Centre, Haywood Hospital, High Lane, Burslem, Stoke-on-Trent, ST6 7AG, UK and 3 University Hospital of North Staffordshire, London Road, Stoke-on-Trent, ST4 6QG, UK

Received: 5 February 2010 Accepted: 8 April 2010

Published: 8 April 2010

\section{References}

1. Woolf AD, Pfleger B: Burden of major musculoskeletal conditions. Bull World Health Organ 2003, 81:646-656.

2. Jordan K, Clarke AM, Symmons DPM, Fleming D, Porcheret M, Kadam UT, et al:: Measuring disease prevalence: a comparison of musculoskeletal disease using four general practice consultation databases. British Journal of General Practice 2007, 57:7-14.

3. Department of Health: The Musculoskeletal Services Framework. 2006.

4. Department of Health: Our health, our care, our say: a new direction for community services. 2006.

5. Dixey J, Bamji A: What is a rheumatologist for? Rheumatology (Oxford) 2007, 46:377-378.

6. Conner-Spady BL, Johnston GH, Sanmartin C, MCGurran JJ, Noseworthy TW: A bird can't fly on one wing: patient views on waiting for hip and knee replacement surgery. Health Expect 2007, 10:108-116.

7. Fortin PR, Clarke AE, Joseph L, Liang MH, Tanzer M, Ferland D, et al: Outcomes of total hip and knee replacement: preoperative functional status predicts outcomes at six months after surgery. Arthritis Rheum 1999, 42:1722-1728.

8. Fortin PR, Penrod JR, Clarke AE, St-Pierre Y, Joseph L, Belisle P, et al.: Timing of total joint replacement affects clinical outcomes among patients with osteoarthritis of the hip or knee. Arthritis Rheum 2002, 46:3327-3330.

9. Speed CA, Crisp AJ: Referrals to hospital-based rheumatology and orthopaedic services: seeking direction. Rheumatology (Oxford) 2005, 44:469-471.

10. Dawson J, Fitzpatrick R, Murray D, Carr A: Questionnaire on the perceptions of patients about total knee replacement. J Bone Joint Surg Br 1998, 80:63-69.

11. Conner-Spady BL, Arnett G, McGurran JJ, Noseworthy TW: Prioritization of patients on scheduled waiting lists: validation of a scoring system for hip and knee arthroplasty. Can J Surg 2004, 47:39-46.

12. Ebinesan AD, Sarai BS, Walley G, Bridgman S, Maffulli N: Total knee arthroplasty: good agreement of clinical severity scores between patients and consultants. BMC Musculoskelet Disord 2006, 7:61.

13. Ayis $S$, Dieppe $P$ : The natural history of disability and its determinants in adults with lower limb musculoskeletal pain. J Rheumatol 2009, 36:583-591.

14. Linsell L, Dawson J, Zondervan K, Rose P, Randall T, Fitzpatrick R, et al.: Prevalence and incidence of adults consulting for shoulder conditions in UK primary care; patterns of diagnosis and referral. Rheumatology (Oxford) 2006, 45:215-221.

15. Badcock L, Lewis M, Hay EM, Croft PR: Consultation and the outcome of shoulder-neck pain: a cohort study in the population. J Rheumatol 2003, 30:2694-2699.

16. Ryall C, Coggon D, Peveler R, Poole J, Palmer KT: A prospective cohort study of arm pain in primary care and physiotherapy - prognostic determinants. Rheumatology (Oxford) 2007, 46:508-515.

17. Thomas E, Croft PR, Paterson SM, Dziedzic K, Hay EM: What influences participants' treatment preference and can it influence outcome? Results from a primary care-based randomised trial for shoulder pain. BrJ Gen Pract 2004, 54:93-96. 
18. Dieppe P, Cushnaghan J, Tucker M, Browning S, Shepstone L: The Bristol 'OA500 study': progression and impact of the disease after 8 years. Osteoarthritis Cartilage 2000, 8:63-68.

19. Katz JN, Solomon DH, Schaffer JL, Horsky J, Burdick E, Bates DW: Outcomes of care and resource utilization among patients with knee or shoulder disorders treated by general internists, rheumatologists, or orthopedic surgeons. Am J Med 2000, 108:28-35.

20. Pace AV, Dowson CM, Dawes PT: Self-referral of symptoms (SOS) followup system of appointments for patients with uncertain diagnoses in rheumatology out-patients. Rheumatology (Oxford) 2006, 45:201-203.

21. Sullivan FM, Hoare TM: New referrals to rheumatology clinics - why do they keep coming back? Br J Rheumatol 1990, 29:53-57.

22. Solomon DH, Bates DW, Schaffer JL, Horsky J, Burdick E, Katz JN: Referrals for musculoskeletal disorders: patterns, predictors, and outcomes. J Rheumatol 2001, 28:2090-2095.

23. Sullivan FM, Hoare T, Gilmour H: Outpatient clinic referrals and their outcome. BrJ Gen Pract 1992, 42:111-115.

24. Armstrong D, Fry J, Armstrong P: Doctors' perceptions of pressure from patients for referral. BMJ 1991, 302:1186-1188.

25. Lack A, Edwards RT, Boland A: Weights for waits: lessons from Salisbury. $J$ Health Serv Res Policy 2000, 5:83-8.

26. Kipping R, Robert G, McLeod H, Clark J: A review of priority scoring and slot systems for elective surgery. School of Public Policy, Health Services Management Centre, University of Birmingham; 2002.

27. Mallen CD, Peat G, Thomas E, Wathall S, Whitehurst T, Clements C, et al:: The assessment of the prognosis of musculoskeletal conditions in older adults presenting to general practice: a research protocol. $B M C$ Musculoskelet Disord 2006, 7:84.

28. Hill JC, Dunn KM, Lewis M, Mullis R, Main CJ, Foster NE, et al:: A primary care back pain screening tool: identifying patient subgroups for initial treatment. Arthritis Rheum 2008, 59:632-641.

29. NHS Information Authority: The Clinical Terms Version 3 (The Read codes). Birmingham: NHS Information Authority; 2000.

30. Dunn KM, Jordan K, Lacey RJ, Shapley M, Jinks C: Patterns of consent in epidemiologic research: evidence from over 25,000 responders. Am $J$ Epidemiol 2004, 159:1087-1094.

31. The EuroQol Group: EuroQol - a new facility for the measurement of health-related quality of life. Health Policy 1990, 16:199-208.

32. Ware JE Jr, Sherbourne CD: The MOS 36-item short-form health survey (SF-36). I. Conceptual framework and item selection. Med Care 1992, 30:473-483.

33. Zigmond AS, Snaith RP: The hospital anxiety and depression scale. Acta Psychiatr Scand 1983, 67:361-370.

34. Arrol B, Khin N, Kerse N: Screening for depression in primary care with two verbally asked questions: a cross-sectional study. BMJ 2003, 327:1144-1146.

35. Von Korff M, Ormel J, Keefe FJ, Dworkin SF: Grading the severity of chronic pain. Pain 1992, 50:133-149.

36. de Vet HC, Heymans MW, Dunn KM, Pope DP, Beek AJ van der, Macfarlane GJ, et al.: Episodes of low back pain: a proposal for uniform definitions to be used in research. Spine (Phila Pa 1976) 2002, 27:2409-2416.

37. Bellamy N: WOMAC Osteoarthritis Index. A User's Guide. London (Ontario): London Health Services Centre, McMaster University; 1996.

38. Koopman C, Pelletier KR, Murray JF, Sharda CE, Berger ML, Turpin RS, et al: Stanford presenteeism scale: health status and employee productivity. J Occup Environ Med 2002, 44:14-20.

Pre-publication history

The pre-publication history for this paper can be accessed here: http://www.biomedcentral.com/1471-2474/11/67/prepub

doi: 10.1186/1471-2474-11-67

Cite this article as: Roddy et al., The Staffordshire Arthritis, Musculoskeletal, and Back Assessment (SAMBA) Study: a prospective observational study of patient outcome following referral to a primary-secondary care musculoskeletal interface service BMC Musculoskeletal Disorders 2010, 11:67

\section{Submit your next manuscript to BioMed Central} and take full advantage of:

- Convenient online submission

- Thorough peer review

- No space constraints or color figure charges

- Immediate publication on acceptance

- Inclusion in PubMed, CAS, Scopus and Google Scholar

- Research which is freely available for redistribution

Submit your manuscript at www.biomedcentral.com/submit
C Biomed Central 